physical and chemical workings of the individual systems of the human body, and have supplied us with various measures by which these can be tested. But of the workings of the human machine as a whole under the varying conditions and stresses of industrial and other forms of activity comparatively little was known. Early in the war it was recognized that the methods of medical examination of recruits then in vogue were far from satisfactory, and their inefficiency was frequently shown up on the parade ground and in the training camps. Especially was this so in tho case of individuals in the Royal Air Force, where it was soon recognized that perfectly " sound" men, in the usually accepted medical sense, might nevertheless be quite unfitted to control an aeroplane. New and more delicate tests were required of the functional activity of the various systems, especially the visual, circulatory, and nervous systems, and of their quickness of response to the constantly changing conditions met with in flying. The medical officers of the Royal Air Force have shown most praiseworthy ingenuity in devising methods for the more efficient testing of the body functions, and have given a great impetus to the study of the healthy human machine at work under conditions hitherto unknown. In the field of industry it is becoming more generally recognized that the test of health is the capacity to perform effective work, and researches are constantly being made into the effects of environmental factors-for example, fatigue, monotony, noise, high illumination-upon the productive efficiency of the industrial worker. It has been shown that the distribution of industrial accidents amongst workers exposed to similar conditions of risk is not a mere matter of chance, but that rach has an individual proneness to accident which can be fairly exactly estimated by testing the rapidity and acruracy with whicin he ressts to certain visual, auditory, and tactile stimuli. Though much still remains to be accomplished, the valuable information thus acquired has already brought about vast improvements in working conditions in many of our industries, and in the standard of health and the productive capacity of those employed therein.

Of modern surgery's debt to the other ancillaty sciences that to bacteriology is assuredly the most fundamental, since it was Pasteur's discovery that fermentation and putrefaction were caused by minute living organisms and were, therefore, vital and not merely chemical pro- cesses, as hitherto believed, that led Lister to his great conception of the antiseptic treatment of that putrefaction then found in almost all wounds-a conception which constitutes, as we have seen, the very starting point of modern surgery. Time permits me merely to mention other advances in bacteriology: the detection of the causative germs of almost all the infective diseases; the enunciation of the great principles of immunity, both " active" and "passive"; the preparation of vaccines and of antitoxic; serums and their use in the prevention and treatment of bacterial infections; the therapy of systemic infections by the intravenous introdurtion of chemical antiseptics resulting, among other notable gains, in that brilliant conquest of the former scourge of syphilis that has been achieved within the present century-all of which constitute milestones along the highway of bacteriology's triumphal progress.

Nor have I time to refer to the great help that modern surgery has received from research workers in anatomy, pathology, and pharmacology, valuable as their contributions have been; nor yet to more general sources of assistance such as the greatly improved means of rapid intercommunication-the telephone, the wireless-and of rapid transport - the motor-car, the aeroplane-factors which have so materially facilitated the prompt application of surgical relief in cases of emergency, whether on land or at sea.

I trust, however, that enough has been said to indicate that modern surgery, with the aid of its allies, has mado and is still making great progress towards that end we all so fervently desire - the prevention of those abnormal conditions, the late results of which to-day necessitate so much surgical attention of a destructive rather than a constructive character. Much yet remains to be done, and the natural sciences must continue to advance, each ready to assist and to accept assistance from its fellows, each along its own special path yet all in mutual fricndly alliance working for the common good, all in zealous pursuit of that goal, unattainable though it must ever remain, that will-o'-the-wisp of perfect lnowledge and complete understanding. In so doing they will but follow the precept and the spirit of that motto upon the crest of our beloved University, in whose venerable precincts this great Association will to-morrow enter upon its full scientific programme, and of which we in this city are all so intensely proud: "Arduus ad solem."- "leaping upwards to the sunshine "- " ever striving towards tho very highest."

\section{PHYSIOLOGY THE BASIS OF TREATMENT.*} BY

\author{
W. E. DIXON, M.A., M.D., F.R.S.,
}

READER IN PHARMACOLOGY, UNIVERSITY OF CAMBRIDGE.

The ultimate aim of medicine is the prevention or cure of disease: this practical aspect so far dominates all other's that it is often referred to as the healing art; indeed, it is difficult to think of medicine apart from treatment.

The term "physiology" is usually used to designate the science of function, whether it is studied in broad outline and dealing with the mechanism of action or as the physico-chemical meclianisns leading up to this action. Disease means the unusual functioning of tissues which may be the result of accident, hereditary weakness, or parasitic organisms. Generally it is wrong to speak of this as malfunctioning: the unusual functioning is physiological and perhaps the best for the organism under the unusual conditions. The science of medicine, then, is nothing more than trained and organized common sense based on physiology. It is still usual to speak of it as an inexact science; this is obviously wrong, since medicine uses the same methods as every other science, and the results of observation are as definite as those of the chemist or physicist; although it is true that, in the complexity of

- Abstract of Presidential Address delivered on July 24th at Capetown before the Section of Plysiology, British Aswociation. the problem with which the physician may have to deal, all the conditions of importance may not be known, and the results of an investigation, though correct for the conditions under which it is undertaken, may be misleading.

When the sciences of physiology and pathology a century ago passed from the realms of natural history to deduction and experiment, they naturally attracted the more original and eager minds in medicine, and the text of the writings of the nineteenth century deals with changes in structure and function. Treatment became neglected, the old shibboleths and rituals of treatment which had held sway for centuries were discarded, and there was nothing with which to replace them. In the middle of the last century $\mathrm{S}$. Skoda and K. Rokitansky perfected a system of physical diagnosis which has had a practical bearing on medicine ever since. Skoda made many experiments with drugs on the patient without any expectation of producing benefit; the patients were not improved and Skoda thought it mattered little how the patient was treated. Scientific medicine looked askance at treatment; textbooks spent many pages in describing the symptoms, diagnosis, and pathology of discase, but two or three lines dismissed the treatment: and even in our times the "scientific" physician is apt to be a diagnostician rather than a healer. The study of disease as an entity was the object aimed at, and a complete case was one which went to autopsy. This state of affairs was unavoidable; there was no specific treatment, there was no science of treatment, for such a science could only come into existence when physiology and pathology had reached some degree of 
precision. Diagnosis was then, and is now, far ahead of treatment; diagnosis is often accurate where there is no satisfactory treatment, and ret diagnosis is only a means to the end.

The science of treatment or pharmacologr is the climax of physiology and pathology, devised to subserve a practical end, and forms an important part of the great biological topic of the influence of conditions on the living organism. Few drugs now exist the mode of action of which is not understood, and the goal is not so far distant when it will be possible to introduce into the animal economy a factor which will exaggerate or retard the function of any tissue or collection of cells in the body, leaving the others unaffected; and most of these results have been obtained by the methods used in expcrimental physiology.

The first object of science is to ascertain facts: certain facts in physiology are relatively easily ascertained-those, for example, which involve the behaviour of ferments of isolated cells or of tissues and which require well-known chemical or physical methods. Other facts involving the physiology of the whole organism are more difficult to interpret, though they are the basis of the therapeutic side of medicine. More and more is physiology being regarded as the application of physics and chemistry to the phenomena of life. The modern attitude is expressed by a distinguished young biochemist who, in reviewing a wellknown book on chemotherapy in 1928, asks, "Might not the time and resources spent on chemotherapeutic research be diverted more profitably to the study of chemical and physical mechanism?" The same attitude is reflected in the awards of fellowships and scholarships for medical research. Formerly all the recipients were primarily. biologists with a medical training; now a medico-biological training is unusual. Physiology in the broad sense in which it was used by Claude Bernard and Huxley has given place to a new physiology of physico-chemical reactions: I might go beyond this and say that physiology is getting further and further from practical medicine, and this is the more regrettable as most of the chairs in physiology are connected with the medical schools and because the science of treatment is largely dependent on experimental physiology.

\section{Pharmacorogy and Therapeutics.}

No branch of experimental biology has received less consideration in Great Britain than that of pharmacology: it is also the most neglected branch of medicine, and although the object of medicine is the healing of the sick; it is amazing that medical schools in Britain, often equipped with all other modern laboratories, lack departments of therapeutics. I was once asked at a meeting, by a leading medical man, what has pharmacology ever done? The answer is, of course, that it has formulated and brought reason and knowledge into treatment of the sick; so much did it impress that great pathologist Ehrlich that he left his serums and turned his attention to drugs, and with the unlimited resources at his disposal gave the world, amongst other drugs, salvarsan; so much did it impress the brilliant French chemist M. Fourneau that he has confined his studies to those of drugs, a study which has resulted in the synthesis of many valuable arsenical compounds and dyes.

I have heard it said by a leading official of our Ministry of Health, speaking to panel practitioners, that they, in the Ministry, do not want stereotyped prescribing in treatment. Surely there was never such nonsense. If there is a best treatment, let us have it, whether it is stereotyped or not. In this respect the British Medical Association has given the profession a lead, and shown by the experience of a great number of doctors that there is a best way of treating varicose ulcer in which the patient gets well quicker than by other ways, an advantage both to the patient and to the community who have to keep him whilst he is unfit for work.

The British pinysician is a skilled diagnostician and is in the forefront in all that pertains to this subject. The literature is so extensive that it is impossible for the arerage clinician to do more than this. But how few of them do, or perhaps the more correct word is "can," occupy the same time in the study of recent advances in general therapeutics! The successful. physician has, generally speaking, to be content with such references to treatment as are to be found in clinical reading. The general physician must always be a necessity for diagnosis; but the details of treatment of patients will, I belicve, in the future be handed over to those who have made a special study of the treatment of that particular group of diseascs from one of which the patient is suffering.

It has been often said that the action of remedies may be best determined by experimenting with them on healthy men. This is not true; quinine is used to treat malaria, yet nct one of the subjective symptoms induced in man has the remotest connexion with its curative properties. The same is true of the use of the iodides in syphilis and salicylates in rheumatism. The experiments of Joig and his pupils in 1825 with camphor, digitalis, and other drugs on healthy men addet nothing of value to pharmacology. Subjective sensations are, it is true, produced, which are erroneously attributed to the drug which has been taken. The late Dr. Rivers and myself were nearly the dupes of such an experiment, which I will give in full becanse it illustrates the imaginary sensations and effects produced by $\mathbf{S}$. Hahnemann and his pupils, by Perkin with his retractors, and by more modern physicians with their mystic apparatus.

Our experiments were made on healthy men under a regular regime as regarded sleep, exercise, and diet. The men were practised with the use of the ergograph during several weeks at the same hour daily, until their output of. work was constant. We found that the administration of a dose of caffeine dissolved in water one hour before the experiment greatly increased the output of work for that day. This was repeated on several occasions, always with the same result, and we naturally regarded the effect as due to the caffeine. This, however, was not the case : the effect was due to the ritual of taking a drug; the drug day assumed an enhanced importance in the mind of the opcrator, and the mental effect sometimes referred to as suggestion was principally responsible. We had no difficulty in showing that water made bitter with a trace of quassia or other simple bitter had a similar effect.

Few, if any, experiments made on man without the most careful controls are of any real value. Properly controlled experiments have been made, however, with many substances. Precise experiments, for example, have been made in both Germany and America with bromides in epilepsy. In these experiments half the epileptics were given potassium chloride and the other half sodium bromide; after several weeks' use the bromide had decreased the number of attacks, whilst the chloride had no distinct action.

\section{Chemotherapy.}

At one time hopes ran high that the chemical structure of the molecule might indicate pharmacological action. During the last fifty years many laborious researches have bcen conducted with this object: to modify the molecule that it may conform to some required action. But the mystery renains as mighty as ever. It is most probable that subtle energy factors binding the molecule-factors not displayed in a formula-control the action; certain it is that drug action is not determined directly by chemical combination with body constituents, but rather by delicate physical processes such as those of adsorption, solution, and surface tension. Chemists have as yet not even determinerl the requirements of the molecule for the production of colour sensation. On the other hand, slight alteration of a molecule already complicated and with a known action has led to the production of many useful compounds, and not infrequently we may foresee the type of action which will occur under such special conditions. Considerations of this nature have led to the synthesis of the new local anaesthetics, antiseptics, antipyretics, diuretics, tropeines, and other useful substances.

But chemistry has taken yct a further step in its assistance given to nedicine in the development of that branch of science to which the name chemotherapy has been fiven. Ehrlich noticed that colouring matters injected into the living organism had a selective sfinity for cortain cells, and he believed that it might be possible, by making use of 
this property, to select suitable substances which would destroy the causal agents of disease-parasites and microbes -and leave the tissues of the host uninfluenced. Parasites calising disease in man nay be crudely divided into worms, protozoa, and bacteria. Chemotherapy-that is, specific therapy of infectious disease-has had marked success in (ruing disease due to parasites in the first and second of these groups; these diseases are found mainly in the tropics. It has obtained much less success in the third group.

Diseases due to protozoa have a special significance in Africa, and it is appropriate that in this meeting some reference should be made to that area of tropical Africa occupring more than a million square niles in which one form of these - namely, trypanosomes - produce their ravages. Two forms of trypanosomes are known to infect man, T. gambiense and T. rhodesiense; the latter is more rapidly fatal than $T$. gambiense, and its treatment is less satisfactory. T. gambiense is transferred by Glossina malpalis, 'T'. brucei by $G$. morsitans and pallidipes, and $T$. rhodesiense by $G$. morsitans. Besides the human form of the discase another tsetse fly is responsible for the trupanosomes of domestic animals, including horses and (attle, T. brucei, which produces the disease n'gana, so that in infected districts draft animals and dairy cattle cannot exist. Trrpanosomiasis is one of the most serious of all tropical diseases, and affects both man and heast; it is a scourge which renders vast tracts of land practically uninhabitable, and which takes its death toll even in thousands, and occasionally even in hundreds of thousands, and ret it is a disease which I believe should be curable, if not preventable.

The members of two groups of chemical substances excel all other's in their curative value in trypanosomiasis and spirochaetosis; these are the organic arsenical compounds and the dyes. The specific action of organic arsenic compounds really begins with some observations of Thomas on the action of atoxyl on trypanosomes. Ehrlich had previously discarded this substance because it was without direct action on the protozoon, but later he observed, like Ievaditi and Mesnil, that in infected animals it had a more pronounced action than that of any other substance up to then employed. Atoxyl was, however; soon discarded as a curative remedy, because it caused permanent and complete blindness, in some cases. Acetyl-atoxyl, known as arsacetin, which was at one time widely used, had no better fortune, and several cases of permanent blindness resulted from its use. Ehrlich's experiments with Hata, in which innumerable arsenical compounds were employed, led him to select salvarsan as the best; in this substance the nitrogen and arsenic are in the meta position, and not in the para, as in atoxyl. This substance as a treatment of syphilis and other spirochaetal infections stands as firmly to-day as it did ten years ago; it has one drawback-it does not influence the condition of the patient if the central nervous system is attacked. Other substances, like tetramethyl hexamino-arsenobenzene (arsalyte Giemsa), have been produced which are also very efficient in sjirochaetosis.

The most satisfactory arsenic compound vet discovered for the cure of trypanosomiasis is tryparsamide. It is less toxic than atoxyl, and has a slightly higher therapeutic index; it has a most marked trypanosidal action in animals, and has been used with some success in cases of sleeping sickness from T. gambiense. One injection causes the disappearance of the parasite from the blood of man, and if the injections are repeated in courses the cure may be complete. Like atoxyl it affects the eye: even the smaller therapeutic doses cause visual disturbance, and the risk of complete blindress is always present; perhaps as many as 30 per cent. of all patients treated with this drug suffer from some eve lesions. Tryparsamide is valuable also in certain forms of syphilis, particularly cerebral syphilis: approximately 40 per cent. of the cases of general paresis committed to the State Insane Hospitals in Wisconsin were restored to sanity (Loevenhart). This action is probably indirect, since there is no evidence to show that it is absorbed into the central nervous system more than other organic arsenicals.

All these organic arsenical compounds must be injecterl in order to produce a satisfactory effect; but one compound, m. amino-p. hydroxyl phenyl arsenic acid, acts upon and destroys spirochaetes when taken by the mouth (Levaditi); it is generally administered as its acetyl derivative, which is known as stovarsol. Stovarsol has been largely used as a preventive to syphilis, but it is now known that it has a remarkable curative action in amoebic dysentery like emetine ( $V$ alenti), and that in cases of benign tertiary malaria it checks the attacks and prevents the returin of the disease, at all events for many months.

It was at first thought that, as laboratory animals are so easily infected with trypanosomes, it should be an easy matter to determine which compounds were likely to be most valuable in the treatment of trypanosomiasis. Unfortunately this is not the case; a drug may cure trypanesomiasis in one animal and not another, and the crucial tests must always be made on man.

Most of the antimony derivatives corresponding with the organic arsenicals have been prepared; for example, that corresponding with acetyl-atoxyl, also $\mathrm{m}$. chloro-p-acetyl amino phenyl stibamate of soda (Heyden 471) have been extensively employed in kala-azar and bilharzia. Speaking. generally, they have a more powerful action than tartar. emetic on such diseases as bilharziasis, kala-azai, and filariasis, and to some of them, like sodium antimony thioglycollate, the parasites do not become readily immune. Unfortunately the organic compounds of antimony have a toxic action on the tissues, and are very difficult to administer, so that antimony tartrate or stihamine urea, a compound which has recently been prepared pure, are generally preferred.

\section{Dyes.}

Many dye substances have been used in medicine: as they are readily adsorbed on to cell surfaces the concentration here is always high, and the surface properties of such cells are often modified in consequence. One general principle which follows from this is that widely different dyes often possess properties in common-for exaniple, that of being antiseptic. To-day I propose to refer only to one group, the benzidine dyes.

Trypan red and trypan blue belong to this group. Trypan blue was employed by my colleague Professor Nuttall in piroplasma infections in animals, with results that most South Africans are well acquainted with. Afridol violet, a derivative of diamino diphenyl urea, and some allied dyes have also a powerful action on piroplasma. How these substances act is not known, for like the organic arsenicals they do not kill the parasite in vitro. They have the property, however, of being adsorbed to the blepharoplast of the trypanosome; this adsorption is associated with diminished virulence of the parasite in infected animals; and after successive inoculations through several animals the organ may disappear. This direct action of a drug on a tissue, causing ultimately the complete disappearance of that tissue, is so remarkable that it is worthy of notice, as it represents the first known action of the kind. The most valuable member of the africlolviolet group so far produced was first made in the Bayer laboratory, but its composition was kept secret. It was, however, subsequently synthesized and its formula published by Fourneau, but only after long trials and infinite patience. Fourneau has made many allied substances and derivatives of " 205 "; the number of such derivatives is obviously legion, and this makes it the more remarkable that he should have succeeded in synthesizing "307," which at the present time is superior as a therapeutic agent to all other dyes in trypanosomiasis. This. " 307 ", has a remarkable action on trypanosomes in laboratory arimals, being 300 times more effective than atoxyl. Its discover $\mathrm{r}$ has also opened a new era in therapeutics, sinco it represents the first chemical substance, which, when administered to man or animals in an infected trypanosome district, gives a complete immunity to the disease for several months; it does not necessarily prevent tripantssome infection, but it prevents the effects of the discase. There is much in these experiments that suggests that we are on the fringe of a new pathologr, and that our present crude methods of preparing antibodies in the future will be replaced by those of the organic and colloid chemist. 
In man " 205 " has not done all that was expected of it; it invariably benefits those that show infection and enlargement of glands-that is, during the early stages of the disease-and its value is also assured even when the nervous system is affected. If care is taken in the treatment, and a series of injections given at not too frequent intervals to prevent the parasite acquiring a tolerance, and if the patient is kept under observation for a prolonged period of time, the happiest issue may be anticipated; " 307 " is much less valuable on n'gana in animals.

\section{Bacterial Infections.}

Much the most important industrial discase in South Africa is the silicosis produced in the extraction of gold from the conglomerate, both the pebbles and the matrix consisting of quartz. The gravity of pnenmoconiosis depends largely on superadded tuberculous infection, to which the workers have a predisposition, and in this respect crystalline silica is much more harmful than either anorphous silicis or carbon.

The report of the Miners' Phthisis Medical Bureau, dealing with the years 1914-26, shows that the incidence of silicosis is increasing. Of the average number of 178,000 natives employed on the scheduled mines during the year 1925-26, of whom 133,260 were employed underground, simple tuberculosis was found to be present in 566 , simple silicosis in 231, and tuberculosis with silicosis in 446. Workers employed in other dusty trades, such as the preparation of asbestos materials, also suffer from pulmonary disability, and Dr. Collis found that five deaths from phthisis had occurred in five years amongst a staff of less than forty workers employed in a factory where asbestos was woven; asbestos contains about 50 per cent. of silica. The dust associated with the process of carding, before its extraction was efficient, at one time in England produced a pneumoconiosis resembling that of silicosis. It is well recognized that a patient suffering from tuberculosis who is placed under treatment will, for a time at least, improve in health, no matter what drugs, vaccines, or "specialties" may be employed. The beneficial result is due, in this as in other disease, to efficient nursing, to the regulation of food, exercise, and sleep, and to light and fresh air. General hygienic measures are of primary importance in treatment, and it is not until all the beneficial effects which are known to ensue from these have bcen exhausted that the physician has any right to ascribe an effect, beneficial or otherwise, to a special treatment.

Drugs are employed in tuberculosis cither with the object of attacking and preventing the growth of the tubercle bacillus or other organisms with which the disease may be associated, of neutralizing poisonous toxins, or of removing or relieving symptoms. It is with the first group that $\mathbf{I}$ am now concerned. Two groups of organic compounds are especially remarkable for their chemotherapeutic action on bacteria. The first group has the quinine complex: the action of optoquin on the pneumococcus and of vuzin on 3acillus diphtheriae is highly specific; the higher and lower homologues have a greatly diminished effect. Many substances destroy bacteria in the test tube, but these drugs act in the animal body as well as in the test tube, and enough can be given by medicinal doses to animals and men to render the blood of these animals bactericidal.

The second group of drugs which exert a marked action on bacteria are certain derivatives of acridine. Trypaflavine was used during the war for infected wounds; unlike most antiseptics, it acts better in the presence of protein, but is not sufficiently selective or specific on micro-organisms in the presence of body tissues to be of any real value; it is easily absorbed, and readily causes oedema. Rivanol is a more recent derivative of acridine. Morgenroth cured streptococcic infections by injertions of rivanol under the skin. The injections to be efficient must be made soon after the infection and in the neighbourhood of the inoculated zone; rivanol will not cure a blood infection. Its discovery is, however, a great advance over any substance previously available in this group, and we can anticipate with some confidence in the near future the introduction of other derivatives which will destroy acute infective agents.

Two other chemical substances are worthy of consideration for the remarkable effects accredited to them in tho, treatment of microbial infections-mercurochrome and hexyl-resorcinol. Clinical evidence shows that mercurochrome exerts a curative effect, espreially in the case of streptococcic and staphylococcic infections, and these effects have been repeated in animal experiments. Hrxylresorcinol has a phenol coefficient of 72 , and it renders the urine in which it is excreted germicidal; many reports show that it effects remarkable cures in cystitis and prelitic. Unlike mercurochrome, the action of this substance, at least in the urine, may be direct on micro-organisms.

The chemotherapeutic substances which are known to act on bacteria are without value in tubcreulosis. Thus flavirn and its silver salts do not influence the tuberculous process in living animals, and the same is true of the quinire. derivatives which have been prepared so. far. The destrortion of the tubercle bacillus presents two special difficultics: first in the fatty and protectire envelope surrounding the bacillus, and second in the small blood supply to the tuberculous lesion. Success, howerer, has been claimed for sereral metallic compounds, and I shall confine m. remarks to three of these. It is br no means clear that the results which have bcen obtained br administering gold salts to tuberculous patients or animals are superior to those given by copper salts, which preceled them. "Krysolgan" was introduced by Foldt: it provents tho growth of the tubercle bacillus in cultures in 1 part in $1,000,000$. Nevertheless, animal cxperiments with this substance are not promising, though the clinical results published in Feldt's monograph in 1923 are cortainly both helpful and inspiring.

Another "gold" cure is that recently introduced by Moellgaard. It is a double thiosulphate of gold and sodium. which, although a well-recognized substance, he ralls "sanocrysin." Like"its predecessors, it retards growth of the tubercle bacillus in glycerinated bouillon in such strengths as 1 in $8,000,000$, but how servm added to the cultures affects.its action I have not discovered. Nevertheless, this is a very important point, since such chemotherapeutic substances as exert an undoubted action on bacteria invariably act the better in the presence of the tissue fluids. Moellgard assumes that his gold injections destroy the tubercle bacillus in vivo, and states that doses which are not poisonous to normal animals kill the tuberculous animal by producing a tuberculin shock. This shock begins with an albuminuria and sometimes haematuria; it is followed by toxic myocarditis and pulmonary oedema. There is, however, no clear evidence to prove that the shock in tuberculous animals after an injection of sanocrysin is due to the destruction of tubercle bacilli and the setting free of endotoxins, which act as tuberculin acts on tuberculous animals. Another feature of importance in these experiments is that the cultures used were attenuated. The doses necessary to kill were enormous, and many control animals failed to die or contract severe disease.

Hoyle and I have recently investigated two new types of gold compounds in tuberculosis. One of these is a complex aurous salt of ethylenethiocarbamide with the formula $(\mathrm{Au}, 2 \mathrm{etu}) \mathrm{H}_{2} \mathrm{O}$, where etu represents ethylenethiocarbamide. It was tested for therapeutic efferts on both human and bovine types of infection. For the former, inoculations were made subcutaneously into guinea-pigs with 1-mg. doses of a virulent human strain. All the animals, control and experimental groups, died within a few dars of one another, and all showed characteristic progressive lesions of similar extent. It was found that treatment with the gold compound in bovine diserse in rabbits prolonged lifo about 50 per cent. when compared with controls. We adopted the arbitrary standard that treated animals should survire at least two or three times longer than the arrage length of life of the controls before clinical trial should be proceeded with. In ricw of the wide rariations in individual susceptibility, and the difficulty that this entails in drawing sound pesitive conclusions from a small series of animals, it is absolutcly necessary to exercise the utmost caution before arousing clinical expectations. 
The second compound tested by us was a complex gold derivative of succinimide, prepared by my colleague Sir William Pope. This substance was tested for therapeutic effect in experimental bovine infections in rabbits. In some animals this treatment was supplemented by injections of potassium iodide subcutaneously; in no case have any therapeutic benefits been observed. There has been no increase in the lengths of life of the treated animals, and the type and extent of disease at post-mortem examination in treated and control animals has in every case up to the present been similar.

These compounds are interesting because the one delays death and the other is entirely without action. They may afford a hint as to the lines on which organic chemists should proceed, and perhaps show that gold in the ionic form is desirable.

\section{The Internal Secretions.}

In the last twenty years much evidence has accumulated to show that the glands of internal secretion are responsible for the regulation of growth, of metabolism, and often for our appearance if not for our very character. Exaggeration or diminution in the secretion of one or other of the tissues may induce conditions so decided as to be obvious to everybody, though the effects produced by minor alterations in the co-ordination of the several secretions may not be so evident. Giants and dwarfs, unusual pigmentation and anaemia, disproportion in the growth of the skeleton, such as enlarged hands and face, bulging deer-like eyes or oriental eyes and beards in women are noticeable to everyone; excessive fatness or emaciation, a choleric or bucolic temperament cause no comment, yet may equally arise in the rictim from a want of co-ordination in the internal secretion.

The general outlook and significance of drug therapy was led into new channels when it was revealed that the animal body through these glands elaborates its own drugs, stores them generally at the seat of formation, and doles them out to the tissues to meet the needs of the economy. Some of these drugs are of the nature of alkaloids comparable with those elaborated by plants. It is a remarkable fact that when Nature elaborates a drug in either a plant or an animal, that drug is invariably the ideal drug for producing the action for which it is characteristic. No drug relieves pain like morphine or produces local anaesthesia so well as cocaine; no dra paralyses the parasympathetics so perfectly as atropine or the motor nerves so effectively as curarine; strychnine supersedes all other drugs in exaggerating spinal reflexes, and caffeine in its jemarkable power of stimulating the psychical centres of the brain. Of the animal drugs, adrenaline has a superlative effect on the sympathetic system, pituitary on the uterus, and thyroxin on general metabolism.

\section{The Suprarenal Gland.}

The suprarenal gland is composed of two distinct organs. The medulla elaborates an alkaloid named adrenaline, the action of which corresponds with stimulation of the entire sympathetic system. What exactly its functions may be in the animal economy is not certain; its output under normal conditions is so limited that it can hardly affect the blood pressure and it is not apparently essential for life. There can be little doubt, however, that in moments of excitement adrenaline is liberated in large amounts and that it is responsible for some of the expressions of the emotions. The action of adrenaline under such conditions is to raise the blood pressure by constricting peripheral vessels, to dilate bronchioles, to erect the hair, to increase the blood sugar, to immobilize the alimentary canal, and to facilitate the clotting of blood. Cammon has shown that cats respond to psychical stimulation, such is may be induced by the presence of a dog, after the entire thoracic sympathetic system has been removed; the interpretation must be that in these emotional conditions adrenaline is set free in relatively large amounts.

The expressions of the emotions, such as anger and terror, are to the animals an advantage: the easy breathing, the ready clotting of the blood, the increased circulation may all have their advantages in a fight. The ultimate cause of spasmodic asthma is constriction of the bronchiolar muscle; if during an asthmatic attack the patient is subjected to some sudden terror or other pronounced emotion the attack sometimes promptly ceases, in a manner exactly simulating the way in which a small injection of adrenaline will abort an attack.

In parts of West Africa the calabar bean, Physostigma, was sometimes used, in trial by ordeal, to determine the innocence or guilt of persons accused of witcheraft or other crimes. A normal person, after drinking an infusion of this bean, promptly vomits and gets rid of the poison. In states of emotion, which might well occur in a guilty per'son, the stomach is flaccid and immobile, vomiting does not occur, and the poison is absorbed. The adrenaline takes some part in this inhibition of vomiting as it stops the movements of the stomach. The bean ordinarily induces violent contractions of the stomach which cause reflex vomiting.

Ephedrine is an alkaloid which has been used by the Chinese as a medicine from time immemorial. It is closely related to adrenaline and has an action very similar to it; but ephedrine acts when taken by the mouth, whilo adrenaline is so easily oxidized that it is destroyed when administered in this manner. Adrenaline causes pulmonary congestion by dilatation of the coronaries; ephedrine has no such effect. Ephedrine has proved of great value in the treatment of spasmodic asthma, since oral administration produces prolonged broncho-dilatation.

\section{The Parathyroids.}

Another striking result from experimental work in the field of internal secretion has recently been obtained in the case of the parathyroid glands. Many vague and unsatisfactory statements existed in the older literature as to the functions of these bodies; but now a potent extract of bovine parathyroid glands accurately standardized can be obtained, and precise knowledge of the part these glands play in the animal economy has become possible. Extracts when injected into a variety of animals raise the level of the blood calcium; if the injection is made into an animal that has been previously parathyroidectomized, tetany and the usual fatal outcome are prevented. Repeated or very large doses in normal animals produce a condition with a definite clinical and biochemical picture-a condition of " hypercalcaemia," in which the blood calcium may rise to very high levels and in which a characteristic train of symptoms is found with terminal renal failure. All this work has shown beyond doubt that the parathyioid glands produce a substance which is responsible for controlling the level of blood calcium, and that interference with this function by removal or disease of the glands can be overcome by treatment with the potent extracts now available.

The Ovary.
The gonads present the clearest evidence of the influence which a tissue may exert on metabolism. I will refer only to the ovary. Virchow is reported to have said that all the peculiarities of the body and mind of woman, alt which in the true woman we admire and revere as womanly, are dependent on the ovary. Knauer showed that this organ was intimately connected with oestrus, and that ovarian grafting could at least partly antagonize the effects of spaying. This ovarian action can be produced in both sexes. If a portion of an ovary is grafted into a castrated male animal the mammary glands and teats hypertrophy, the glands develop to the secretory stage, and the animal comes to resemble a pregnant female: males so grafted become hyperfeminine in appearance. In the male the development of the mammary glands is uninterrupted, the Graafian follicles mature but do not rupture, and the ovary soon degenerates. In the engrafted femalo development is slower and, unlike the male, shows a rhythm which is associated with the development of the Graafian follicle, and in the regressive phase with its rupture and the formation of the corpus luteum. 
The ovary does not function till puberty, and there is considerable evidence to show that this is brought about by some internal stimulus. A young ovary grafted into an adult male or female will begin its secretion sooner than its age warrants, whilst an adult ovary engrafted into a young animal will not function until the animal reaches maturity. This fact is of practical importance, since in cases of infantilism it is not necessarily the ovaries which are at fault, and ovarian transplantation may not improve the patient. The ovary differs from other organs of internal secretion in that it functions in a cyclic manner, and it is obvious that extracts made from ovarian tissue may exert a different action according to the period of the cycle when they are made.

Numerous extracts have been prepared from the ovary which are reputed to exert one or other type of action. "Oestrin" is the name given to one such substance; it can be made from many sources, both animal and vegetable, besides the ovary. Oestrin exerts a very dofinite action in lower animals, but its use in man is so variable and disappointing as to make it valueless in practical medicine. When it is injected subcutaneously into spaved rats and mice it produces typical oestrus with normal sex instincts, and when injected into immature animals it induces puberty; regular injections at fixed intervals will keep animals sterile.

Many experimental observations show that the corpus luteum is concerned with the rhythm of the oestrous cycle and with the prevention of ovulation. A persistent corpus luteum, in both animals and women, produces sterility, a condition which is cured by its removal. The presence of fully formed corpora lutea appears to inhibit some ovarian secretion, and this condition obtains in animals for a time between the heat periods, but more particularly during pregnancy. In women there is plenty of pathological cridence to show that functional corpora lutea are not present during menstruation. If the corpora lutea exert this controlling action on ovarian function, then their removal should release the normal ovarian function; such operations during pregnancy are invariably followed by abortion. On the other hand, injections of properly prepared corpus luteum prevent ovulation; this has been shown in the case of the hen, the rabbit, and the guinea-pig.

\section{Interstitial Hormone.}

$A$ third active substance distinct from oestrin and corpus luteum is elaborated from the ovary, and was described by Marshall and myself. The substance is water-soluble and therm-stabile, and can be prepared from the ovary at one stage of its cycle only, by maceration with warm saline, followed by boiling and filtering. The injection of this substance into animals causes a secretion of pituitrin, and this in turn renders the uterus supersensitive and highly responsive to other forms of stimulation.

The pituitary gland is intimately connected with the phenomenon of pregnancy; statistics show that the size and weight of the gland in men and nuHiparae are about the same; in primiparae the weight has increased by about 50 - per cent., and in multiparae by about 90 per cent., though most of the increased weight is due to the anterior lobe. The posterior pituitary substance has at least three actions of importance in medicine: it inhibits the secretion of urine, it has an antagonistic action to the insulin effect, and it sensitizes and, in larger doses, contracts the plain muscle of the uterus. The last action is so profound that it overshadows all the other muscular effects, and pituitrin may be said to have a true specific action on uterine muscle in rendering it supersensitive to every form of extraneous stimulus. Our experiments showed that at one stage only of the ovarian cycle was this hormone elaborated -nameiy, at the stage when the corpora lutea are degenerating. So long as the corpora are functioning they control the metabolism of the ovary, but when they degenerate control is lost and the ovary liberates the specific substance which cxcites the pituitary gland to secrete. This means that extracts of the ovary made between the heat periods or during pregnancy are without effect on the pituitary gland, but extracts made just before the heat period or just before parturition induce secretion of the gland. $\boldsymbol{A}$ s the significant action of pituitary extract is to sensitize the uterus it is difficult, if not impossible, to avoid the conclusion that these two phenomena are closely associated.

It is well known that substances introduced into the cerebro-spinal fiuid find their way almost immediately into the blood, and hence it might be expected that cxtracts made from the blood of pregnant rabbits obtained at the time of delivery would have a contractile influence on the uterus; this has been found to occur. Similarly, blood obtained from pregnant women at the time of delivery contracts the guinea-pig's uterus to a considerably greater degree than normal blood. Mayer collected the cerebrospinal fluid from women during Caesarean section. This fluid he injected subsequently into ten women with deficient labour pains. In eight of the women pains were induced, which in four were followed by the birth of the child. In another case an intradural injection was made, which was followed by labour pains within twenty-four hours. The interaction of bile salts and pituitary secretion is antagonistic on the uterus. Hofbauer shows that thero is a steady increase in the bile salts in the blood of pregnant women as gestation proceeds. He thinks that this factor is responsible for the control of the pituitary secretion during pregnancy, and that towards the end of labour the pituitrin action overshadows the bile-salt action, so that labour occurs.

All these experiments consistently support the view that in the presence of fully formed corpora lutea the normal ovarian secretion is held in abeyance, and this is the condition for a short part of the time between the heat periods, but more particularly during pregnancÿ. At the close of pregnancy, when the corpora lutea are in an advanced stage of involution, the normal secretory activity recurs, and the pituitary gland is excited to secrete more actively. When the threshold stimulus of the pituitrin on the uterus is reached the pains of labour set in and parturition results. The well-known phenomenon of the growing irritability of the uterus in the later stages of pregnancy, which is the typical effect of the pituitary action, is explained as being functionally correlated with the involution of the corpus luteum. It is not suggested that the ovario-pituitary endocrine mechanism is the sole factor in producing labour pains. No doubt the foetus itself acts as a direct stimulus, and without the foetus the intense muscular contractions would not occur, but it is also clear that the onset of labour cannot easily be accounted for without postulating some further exciting cause apart from the foetus and the uterus. No romance can be more remarkable than the fret that doctors, by using pituitary extract to stimulate the uterus in pregnancy, should have adopted the method which Nature herself employs, and that physiological function is, after all, a pharmacological action.

\section{Concluding Remarks.}

I have endeavoured to show that all precise knowledgo in therapeutics is based upon controlled experiments on animals or man, and that the elucidation of the action of medicaments by the methods and data of experimental physiology is one of the most important steps taken to place medicine on a srientific basis. How important this is may be gauged from the fact that all fundamental advances in treatment in the last thirty years have originated, directly or indirectly, from experiments on animals. There can be no doubt, then, that the future of therapentics, and therefore of medicine as a whole, is intimately connected with physiology; there can be no doubt that advance in the practice of medicine is dependent on those trained in the methods and fundamental truths of physiology, who devote themselves in the ward and biological laboratory to investigating how best to prevent or cure disease, and so relieve suffering.

Britain for fifty years has every reason to be proud of her progress and achievements in phrsiology; it is acknowledged that she can show records second to none, and that her savants have included some of the world's greatest investigators. It remains for us to hope that in the futuro she may attain equal success in the associated sciences directly concerned with the relief of suffering and cure of disease. 